DePauw University

Scholarly and Creative Work from DePauw University

\title{
An inward focus of attention during information security decision making: Electrophysiological evidence
}

Robert West

DePauw University, robertwest@depauw.edu

Kate Cowger '22

DePauw University

Follow this and additional works at: https://scholarship.depauw.edu/psyc_facpubs

Part of the Neuroscience and Neurobiology Commons

\section{Recommended Citation}

West, R., \& Cowger, K. (2021). An inward focus of attention during information security decision making: Electrophysiological evidence. In F. D. Davis et al. (Eds.), Information Systems and Neuroscience, 103-111, Lecture Notes in Information Systems and Organisation.

This Conference Proceeding is brought to you for free and open access by the Psychology and Neuroscience at Scholarly and Creative Work from DePauw University. It has been accepted for inclusion in Psychology and Neuroscience Faculty Publications by an authorized administrator of Scholarly and Creative Work from DePauw University. 


\title{
Proceedings NeuroIS Retreat 2021
}

\author{
Virtual Conference | June 1-3 \\ www.NeuroIS.org
}

Fred D. Davis, René Riedl, Jan vom Brocke, Pierre-Majorique Léger, Adriane B. Randolph, Gernot Müller-Putz (Eds.)

The final proceedings will be published by Springer. 


\title{
Preface
}

The proceedings contain papers presented at the 13th annual NeuroIS Retreat held June 1-3 2021. NeuroIS is a field in Information Systems (IS) that uses neuroscience and neurophysiological tools and knowledge to better understand the development, adoption, and impact of information and communication technologies (www.neurois.org).

The NeuroIS Retreat is a leading academic conference for presenting research and development projects at the nexus of IS and neurobiology. This annual conference promotes the development of the NeuroIS field with activities primarily delivered by and for academics, though works often have a professional orientation.

In 2009 the inaugural NeuroIS Retreat was held in Gmunden, Austria. Since then, the NeuroIS community has grown steadily, with subsequent annual Retreats in Gmunden from 2010-2017. Beginning in 2018, the conference is taking place in Vienna, Autria. Due to the Corona crisis, the organizers decided to host a virtual NeuroIS Retreat in 2021.

The NeuroIS Retreat provides a platform for scholars to discuss their studies and exchange ideas. A major goal is to provide feedback for scholars to advance their research papers toward high-quality journal publications. The organizing committee welcomes not only completed research, but also work in progress. The NeuroIS Retreat is known for its informal and constructive workshop atmosphere. Many NeuroIS presentations have evolved into publications in highly regarded academic journals.

This year is the seventh time that we publish the proceedings in the form of an edited volume. A total of 27 research papers were accepted and published in this volume, and we observe diversity in topics, theories, methods, and tools of the contributions in this book. The 2021 keynote presentation entitled "Decision Neuroscience: How it started and where we are today" is given by Antoine Bechara, professor of neuroscience and psychology at the University of Southern California (USC) in Los Angeles, USA. Moreover, Moritz Grosse-Wentrup, professor and head of the Research Group Neuroinformatics at the University of Vienna, Austria, gives a hot topic talk entitled "How (not) to interpret Multivariate Decoding Models in Neuroimaging".

Altogether, we are happy to see the ongoing progress in the NeuroIS field. Also, we can report that the NeuroIS Society, established in 2018 as a non-profit organization, has been developing well. We foresee a prosperous development of NeuroIS.

June 2021

\author{
Fred D. Davis \\ René Riedl \\ Jan vom Brocke \\ Pierre-Majorique Léger \\ Adriane B. Randolph \\ Gernot Müller-Putz
}




\section{Sponsors}

We thank the sponsors of the NeuroIS Retreat 2021:

Main Sponsors
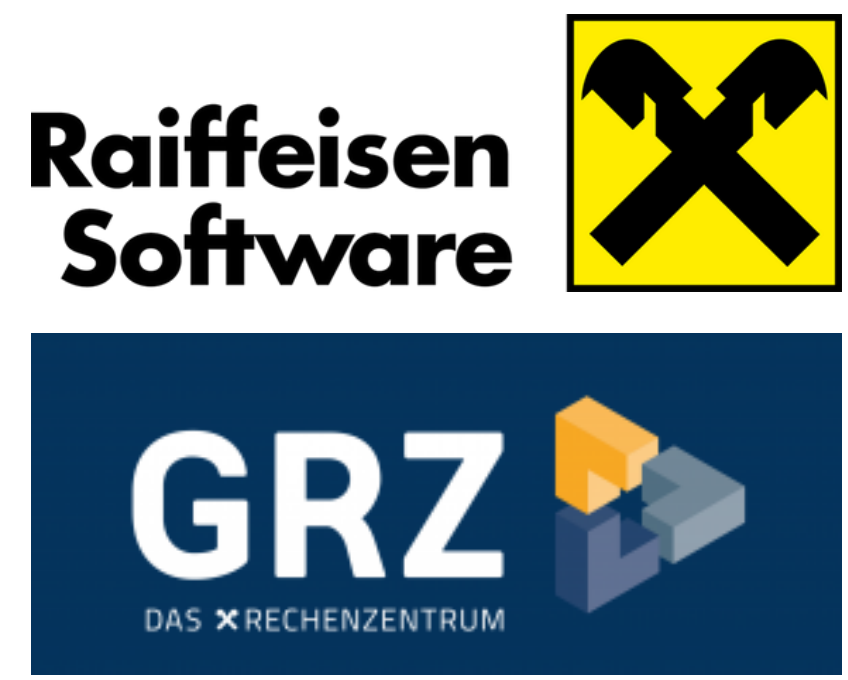

Further Sponsors
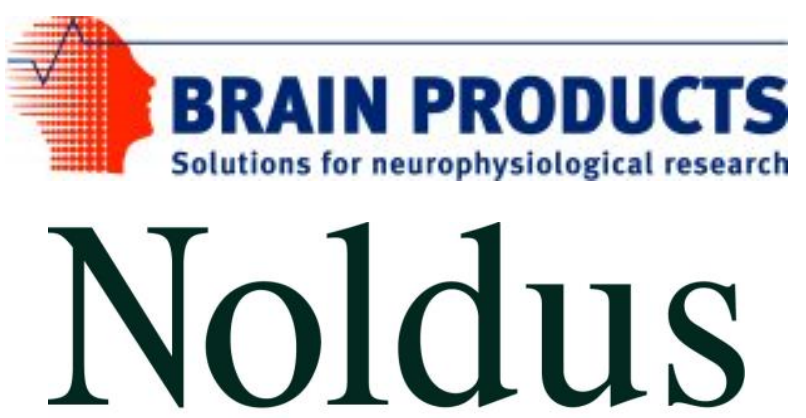

Information Technology 


\title{
Antoine Bechara - Keynote
}

\author{
Decision Neuroscience: How It Started and Where We Are Today
}

Decision neuroscience is an emerging area of research whose goal is to integrate research in neuroscience and behavioral decision-making. Neuroeconomics is a more specialized field of study that seeks to bridge neuroscience research on human choice with economic theory, whereas neuromarketing addresses the neuroscience behind consumers' choices, including product branding, preference, and purchase decisions. More recent research seeks to include the field of information science by examining the impact of social media and other technology use on the human brain. All these areas capitalize on knowledge from the fields of neuroscience, behavioral economics, finances, marketing, and information science to explore the neural "road map" for the physiological processes intervening between knowledge and behavior, and the potential interruptions that lead to a disconnection between what one knows and what one decides to do. Thus, decision neuroscience is the domain that captures the interests of scientists who are attempting to understand the neural basis of judgment and decisionmaking in health as well as social behavior.

\section{Moritz Grosse-Wentrup - Hot Topic Talk}

\section{How (not) to Interpret Multivariate Decoding Models in Neuroimaging}

Multivariate decoding models are replacing traditional univariate statistical tests in the analysis of neuroimaging data. Their interpretation, however, is far from trivial. In this presentation, I outline various pitfalls and discuss under which conditions they can provide insights into the (causal) question of how neuronal activity gives rise to cognition and behavior.

\section{Panel Discussion}

\section{Success Factors in Publishing NeuroIS Research in Top IS Journals: Experiences of MIS Quarterly Editors and Reviewers}

Moderator: Fred D. Davis

Panelists: Ofir Turel, Tony Vance, Adriane B. Randolph, Eric Walden 


\section{Table of Contents}

Where NeuroIS Helps to Understand Human Processing of Text: A Taxonomy for Research Questions Based on Textual Data.

Towards a Psychophysiological Investigation of Perceived Trustworthiness and Risk

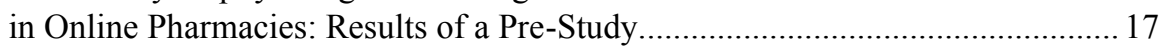

Exploring the Influence of Personality Traits on Affective Customer Experiences in Retailing: Combination of Heart Rate Variability (HRV) and Self-Report Measures

Motor Dysfunction Simulation in Able-Bodied Participants for Usability Evaluation of Assistive Technology: A Research Proposal ....................................................... 39

Exploring the Potential of NeuroIS in the Wild: Opportunities and Challenges of Home Environments 48

Exploring the Recognition of Facial Activities Through Around-the-Ear Electrode Arrays (cEEGrids).

Leveraging NeuroIS Tools to Understand Consumer Interactions with Social Media Content 66

Optimizing Scatterplot-Matrices for Decision-Support: An Experimental EyeTracking Study Assessing Situational Cognitive Load ...................................... 73

“Overloading” Cognitive (Work)Load: What are We Really Measuring? .................. 86

On Electrode Layout in EEG Studies: A Limitation of Consumer-Grade EEG Instruments.

Predicting In-Field Flow Experiences Over Two Weeks from ECG Data: A Case Study

An Inward Focus of Attention During Information Security Decision Making: Electrophysiological Evidence

EyeTC: Attentive Terms and Conditions of Internet-based Services with WebcamBased Eye Tracking

Detecting Flow Experiences in the Field Using Video-Based Head and Face Activity Recognition: A Pilot Study ..... 134

Understanding the Potential of Augmented Reality in Manufacturing Environments 142

On How Mind Wandering Facilitates Creative Incubation While Using Information Technology: A Research Agenda for Robust Triangulation 155

Consumers Prefer Abstract Design in Digital Signage: An Application of Fuzzy-Trace Theory in NeuroIS 164 
Topographic Analysis of Cognitive Load in Tacit Coordination Games Based on Electrophysiological Measurements .............................................................. 180

Active Learning Techniques for Preparing NeuroIS Researchers ........................... 191

Examining the Impact of Social Video Game Tournaments on Gamers' Mental WellBeing 197

Continuing Doctoral Student Training for NeuroIS and EEG During a Pandemic: A Distance Hands-On Learning Syllabus

Design Mode, Color, and Button Shape: A Pilot Study on the Neural Effects of Website Perception

Does Media Richness Influence the User Experience of Chatbots: A Pilot Study ... 225

Development of a New Dynamic Personalised Emotional Baselining Protocol for Human-Computer Interaction

Mediators of the Relationship Between Self-Control and Pathological Technology Use: Negative Affect and Cognitive Failures, But Not Self-Efficacy....

High Fidelity Vibrokinetic Stimulation Augments Emotional Reactivity and Interhemispheric Coherence During Passive Multimedia Interaction.

Explainable Artificial intelligence (XAI): How the Visualization of AI Predictions Affects User Cognitive Load and Confidence 


\title{
An Inward Focus of Attention During Information Security Decision Making: Electrophysiological Evidence
}

\author{
Robert West, and Kate Cowger \\ Department of Psychology and Neuroscience, DePauw University, Greencastle, \\ USA \\ robertwest@depauw.edu,katecowger_2022@depauw.edu
}

\begin{abstract}
Insider threat represents a significant source of violations of information security. Our previous research using event-related potentials (ERPs) has revealed patterns of neural activity that distinguish ethical decision making from decisions that do not involve an ethical component. In the current study, we sought to gain insight into the locus of the effect of ethical decision making on the posterior N2 component of the ERPs. The ERP data revealed that the N2 was greater in amplitude for control trials relative to ethical violation trials, and time-frequency analyses revealed that this resulted from a reduction in phase-locked activity across trials rather than a decrease in EEG power. These findings may indicate that ethical decision making related to information security is associated with a greater inward focus of attention than is the case for decision making on control trials.
\end{abstract}

Keywords: Information security, insider threat, ethical decision making, ERPs

\section{Introduction}

Violations of information security represent a significant threat to the well-being of individual citizens, corporations, and governments. Insider threats (i.e., violations of information security that result from the actions of individuals that are part of an organization) may account for as much as $50 \%$ of instances of violations of information security [1]. This has led to the intense investigation of system and person level variables such as personal norms and ethics, self-efficacy, rewards, detection certainty, etc. that moderate insider threat using a variety of methodologies [2]. In our own research, we have incorporated scalp recorded EEG to examine neural activity related to ethical decision making in the domain of information security [3,4]. These studies reveal modulations of the ERPs that consistently distinguish ethical dilemmas from neutral dilemmas, and are sensitive to individual differences in variables known to predict instances of insider threat (e.g., moral belief and self-control) [5]. In the current study, we build upon our recent research using the Information Security Paradigm (ISP) [3] by examining one possible locus of the effect of ethical violations on the posterior N2 component of the ERPs. Specifically, we considered whether an inward focus of attention driven by the sustained consideration of an ethical dilemma presented in a 
scenario may lead to a reduction in the allocation of attention to visual stimuli including the decision prompt may account for the finding that the N2 is greater in amplitude for control trials than for ethical violation trials.

In the ISP [3], individuals read a set of scenarios and are then presented with a decision prompt. The scenarios differ in terms of whether or not a hypothetical information system specialist is faced with a possible violation of information security (e.g., the unauthorized access of a secure server) or a decision that does not have an ethical component (e.g., assisting a colleague in retrieving a client list). Research incorporating the ISP provides support for a Dual Process Theory of decision making wherein controlled strategic processes related to self-control and more rule-based processes related to moral convictions are associated with different patterns of neural activity (i.e., modulations of the event-related potentials or ERPs)[5]. For instance, low self-control is associated with a reduction in the amplitude of slow wave activity over the lateral frontal region of scalp between 500-1500 ms after the onset of the decision prompt in the ISP. This finding has been interpreted as reflecting a reduced tendency for individuals with low self-control to engage in effortful frontal processes during decision making. In contrast, high moral potency is associated with reduced slow wave activity over the frontal region. A finding that may reflect individuals with high moral potency rejecting violations of information security based upon rule-based moral standards that do not require the utilization of effortful processes.

ERPs recorded after the onset of the decision prompt in the ISP consistently reveal that the amplitude of the posterior N2 component is greater in amplitude for control trials than for violation trials [3-5]. This finding is interesting, as the perceptual characteristics (i.e., intensity, color, number of characters or words) of the prompts is similar across the two types of trials, so it seems unlikely that this effect is stimulus driven. One explanation for the effect on the posterior N2 is that the consideration of ethical violations is associated with a greater inward focus of attention that is be driven by the need to resolve conflict between the benefits (i.e., financial gain) and costs (i.e., being discovered or violating a personal or social norm) of the unethical act relative to when individuals are considering control scenarios that do not involve unethical behavior. This idea is consistent the literature examining the effects of spatial and feature attention on the amplitude of the posterior N2 [6]. This research reveals that the amplitude of the $\mathrm{N} 2$ component is greater when attention is directed to a feature of the external world (e.g., a spatial location or stimulus color) relative to when attention is not directed toward a stimulus. Furthermore, this enhancement effect is also observed in the steady state visual evoked potential. For instance, Anderson and Müller [7] report a selective increase in power at the stimulation frequency for an attended versus unattended color, but not at other frequencies. Work examining the effect of cognitive or memory load is also consistent with this idea. Specifically, the amplitude of the posterior N2 is reduced as working memory load increases and when individuals maintain a delayed intention in the context of prospective memory [8].

Here we report data from a new study designed to examine the locus of the effect of an ethical violation on the posterior N2 using ERP and time-frequency methods. The time-frequency analyses allowed us to explore the locus of the N2 effect in 
the ERPs, determining whether the effect was differentially related to the magnitude of the neural response (i.e., EEG power) or to phase-locking of the EEG across trials to onset of the prompt (i.e., intertrial coherence (ITC)). We also sought to examine the role that emotion may play in decision making in the ISP by having participants rate the intensity of their emotional response to the scenarios and prompts after each trial. Three hypotheses were considered based upon previous ERP studies using the ISP [35], the broader literature relating information processing to measures of EEG using time-frequency methods [6,7], and the literature examining the role of emotion in moral decision making $[9,10]$.

$\mathrm{H} 1$ : The amplitude of the posterior N2 will be greater for control trials than for violation trials, and the amplitude of sustained ERP activity over the right hemisphere will be greater for violation trials than for control trials.

$\mathrm{H} 2$ : ITC at the time of the posterior N2 will be greater for control than for violation trials, if this effect reflects the inward focus of attention for violation trials.

$\mathrm{H} 3$ : Violation trials will be associated with a stronger emotional response than control trials.

\section{Method}

\section{Participants}

Sixty-three students from DePauw University enrolled in introductory and intermediate level Psychology courses participated in the study. Participants were 18-22 years of age; 47 participants identified as female and 15 identified as male; the racial and ethnic distribution was 42 white, 8 black or African American, 8 Asian, 1 Hispanic/Laninx, 2 other, and 1 unidentified; and one participant's demographic information was lost due to an Internet connectivity error.

\section{Materials}

The ISP was adapted from Kirby et al. [4]. The task included two types of scenarios (i.e., control and violation). For each scenario a hypothetical situation was posed involving Josh, an information technology specialist with extensive knowledge of his company's IT systems. For control scenarios, the situation involved a decision to engage in some activity that did not involve an unethical behavior (e.g., assist a colleague with a project). For violation scenarios, an action was described wherein Josh would need to violate the company's information security policy in order to complete the task (e.g., unauthorized access of a secure server). Each trial began with a blank screen $(500 \mathrm{~ms})$ that was followed by the scenario that remained on the screen until participants pressed the space bar. The scenario was replaced by a fixation cross at the center of the screen for $500 \mathrm{~ms}$ followed by the onset of the prompt that remained on the screen until a response key was pressed. Individuals responded to the prompt by pressing $\mathrm{C}$ (left middle finger), $\mathrm{V}$ (left index finger), $\mathrm{B}$ (right index finger), or $\mathrm{N}$ (right middle finger) to indicate their choice (No, Likely No, Likely Yes, Yes, respectively). The prompt was followed by a screen indicating that the individual should enter their "Emotional Response" to the scenario and prompt on a 1-4 scale (Not at all 
strong, Somewhat Strong, Very Strong, Extremely Strong) using the same keys as used to respond to the prompt. A TTL pulse was aligned with the onset of the prompt and delivered to the amplifier to mark the onset and meaning of the prompt in the EEG data stream. There were two practice trials and 16 test trials for each type of scenario. Behavior (choice and response time) and ERPs were measured from onset of the prompt. Prior to beginning the trials, participants were told that Josh was under a lot of financial strain and work-related stress. They were also instructed to go through the task imagining that they were Josh and to answer the prompts from Josh's perspective.

\section{Procedure}

Once individuals had arrived at the lab, each was given a descriptive summary of the procedure that included an introduction to the EEG cap, electrodes, and conductive gel to be placed in their hair. Individuals provided signed informed consent for the study that was approved by the Institutional Review Board of DePauw University. The participants then completed a demographic survey reporting their age, gender, racial and ethnic background, and years of college completed followed by questionnaires that measured individual differences in self-control [3], grit [11], depression [12], Internet addiction [13], and smartphone addiction [14]. These measures were collected to examine how individual differences are related to neural recruitment elicited during ethical decision making. The participants completed the ISP, counting Stroop task, and doors task while EEG was recorded. After these tasks, individuals were debriefed and paid $\$ 5$ in winning for the doors task and received research credit for an introductory or intermediate level psychology as compensation for participation.

\section{EEG Recording and Analysis}

The EEG were recorded with a 32 channel actiCAP and actiCHamp active AG/AGCL electrode system using the Brain Vision Recorder Software (Brain Vision, LLC). The data were sampled at $500 \mathrm{~Hz}$, from DC-150 Hz and digitized at 24 bits. Thirty electrodes were placed in the standard Brain Vision 32 electrode cap configuration (CP5-CP6 were replaced with the ocular electrodes) and two were placed below the eyes to monitor blinks and vertical eye movements. During recording the electrodes were referenced to electrode $\mathrm{Cz}$, and impedances were maintained below $20 \mathrm{~K} \Omega$. For analysis, the average reference was calculated after the ocular artifacts were corrected using ICA.

The EEG data were processed using EEGLAB [15] and ERPLAB [16]. A .1$30 \mathrm{~Hz}$ IIR filter was applied to the EEG; $1-2$ bad electrodes were interpolated as needed; ocular artifacts associated with blinks and saccades were corrected using ICA and visual inspection of the data. ERPs were averaged for -200 to $2000 \mathrm{~ms}$ and the timefrequency analysis included -500 to $2000 \mathrm{~ms}$ around onset of the prompt. For the ERP and time-frequency analyses a permutation t-test was used with 1000 iterations for statistical inference with an uncorrected $p=.01$. The time-frequency analyses used a $500 \mathrm{~ms}$ pre- and post-stimulus buffer, so the epochs for these analyses represent onset of the prompt (time 0) to $1500 \mathrm{~ms}$. 


\section{Results}

\section{Psychometric and Behavioral data}

To establish the reliability of the ISP, we examined internal consistency for the control and violation items using Chronbach's $\alpha$. Both sets of items demonstrated clear internal consistency (control $\alpha=.90,95 \% \mathrm{CI}[.86-.93]$; violation $\alpha=.93$, 95\% CI[.91-.96]) and there was no evidence that dropping any of the items would result in an improvement in reliability. These data indicate that the ISP has good psychometric properties for a research instrument.

The choice data revealed that on average individuals responded between likely no and likely yes for both control $(\mathrm{M}=2.37, \mathrm{SD}=.61)$ and violation $(\mathrm{M}=$ $2.51, \mathrm{SD}=.69$ ) items, and choices responses did not significantly differ between items, $\mathrm{t}(61)=1.81, \mathrm{p}=.075$. Response time was also similar for control $(\mathrm{M}=1.3$ seconds, $\mathrm{SD}=.50)$ and violation $(\mathrm{M}=1.3$ seconds, $\mathrm{SD}=.47)$ items, $\mathrm{t}(61)=.30, \mathrm{p}=$ .77. Relative to previous research with this task [3-5], choice responses are higher for violation items than would be expected and response times are noticeably faster. The reason for the difference in choice behavior between the current sample and prior studies is unclear.

The emotional response data revealed that on average individuals found the control items $(\mathrm{M}=2.82, \mathrm{SD}=.26)$ to elicit a stronger emotional response than the violation items $(\mathrm{M}=1.89, \mathrm{SD}=.55), \mathrm{t}=12.77, \mathrm{p}<.001$. Individuals did take longer to rate the violation items $(\mathrm{M}=2.5$ seconds, $\mathrm{SD}=.80)$ than the control item $(\mathrm{M}=2.3$ seconds, $\mathrm{SD}=.70), \mathrm{t}=3.77, \mathrm{p}<.001$. These data diverge for the moral decisionmaking literature in demonstrating that at least within the ISP ethical decision-making is not associated with heightened emotion.

\section{ERP and Time-frequency data}

Figure 1a includes the ERPs averaged over electrodes for the occipital (O1-Oz-O2) and frontal-central-parietal (FC2-CP2-Cz-Pz) regions. For the occipital region, the ERP data revealed that the amplitude of the posterior N2 was greater for control trials than for violation trials. This finding is consistent with our previous research and provides evidence supporting Hypothesis 1 . The sustained ERP activity over the right frontal-central-parietal regions reflecting greater negativity for violation trials than for control trials between $200-1000 \mathrm{~ms}$ after onset of the prompt is also consistent with the findings of our previous research [3-5], and may reflect longer lasting deliberative processing related to ethical decision making in the task.

Figure 1. a) Grand-averaged ERPs over the occipital region and right hemisphere demonstrating the effect of trial on the N2 and sustained ERP activity over the right hemisphere, b) time-frequency plots for control and violation (Josh) trials, c) ITC plots for control and violation (Josh) trials. 
a)
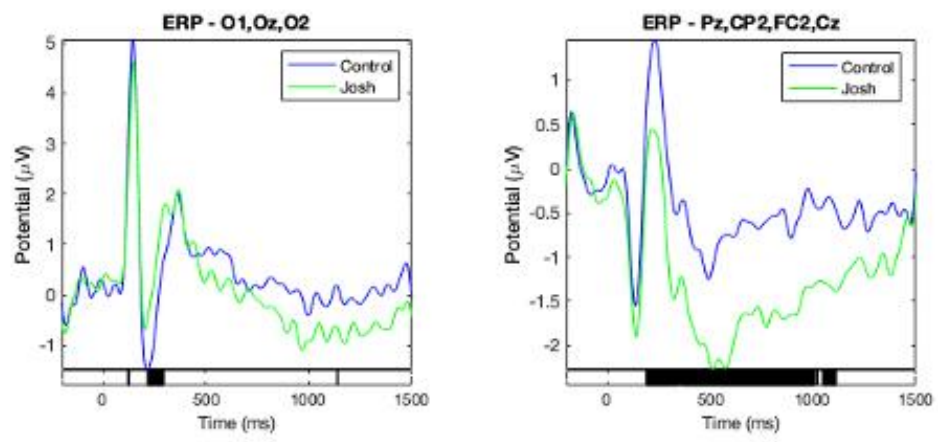

b)
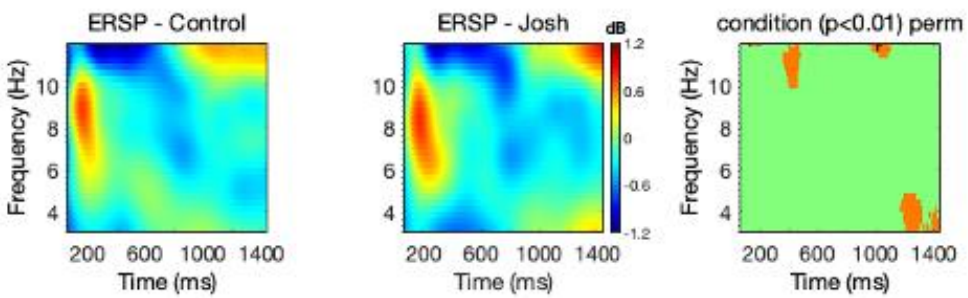

c)
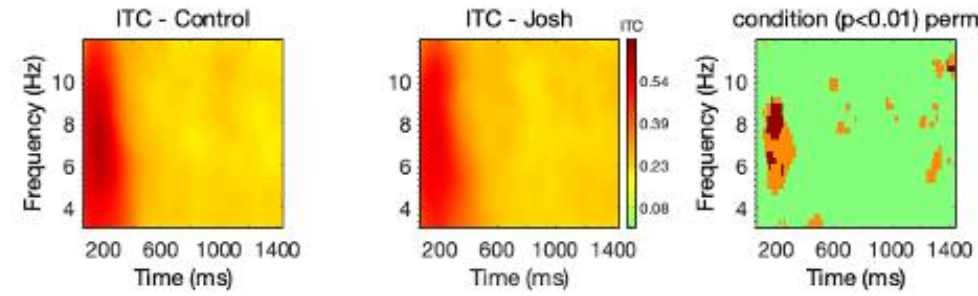

For the occipital region, the time-frequency analysis revealed an increase in power around $200 \mathrm{~ms}$ after onset of the prompt in the Theta/Alpha band $(5-10 \mathrm{~Hz})$ that was followed by suppression in the high Alpha band (Figure 1b). This increase in Theta/Alpha power did not differ between the control and violation trials in the timefrequency data (Figure 1b). The ITC analysis revealed increased coherence around $200 \mathrm{~ms}$ in the Theta/Alpha bands that was greater for control trials than for violation trials (Figure 1c). These findings provide support for Hypothesis 2, indicating that the effect of trial on the posterior N2 arises from differences in phase locking to the prompt across trials rather than differences in the magnitude of the neural response (i.e., power) to the prompt. 


\section{Discussion}

The current data contribute to a growing body of literature that serves to refine our understanding of the psychological and neural correlates of ethical decision making in the ISP [3-4]. The emotion rating data reveal that violation scenarios in the ISP may not elicit strong conscious emotion responses that shape decision making. This finding is interesting within the context of the broader literature related to moral and ethical decision making demonstrating the emotion or affective processes can drive decision making in these domains $[9,10]]$. However, the contribution of emotion to decision making is often considered a System 1 input that may not operate at the level of conscious awareness [9]. Given this, further efforts to assess the implicit influence of emotion in the ISP may be worth exploring. The ERP data also revealed slow wave activity extending from the frontal to parietal region over the right hemisphere. The is consistent with previous research using the ISP [3-5] and indicates that ethical decision making in the task is associated with slow deliberative processes that engage a broadly distributed cortical network.

The findings of the current study provide clear support for our hypotheses related to the locus of the effect of an ethical violation on the amplitude of the posterior $\mathrm{N} 2$. We replicated the effect of trial type on the posterior N2 and demonstrating that this effect is associated with a decrease in phase-locked activity rather than a reduction in EEG power associated with the onset of the prompt for violation trials relative to control trials. These data provide evidence for the idea that considering violations of information security may be associated with a greater inward focus of attention than considering scenarios that do not involve an ethical component. The effect of trial on ITC related to the posterior N2 may provide a foundation to further explore the nature of the effect of individual difference variables such as self-control and moral belief on decision-making processes related to information security $[17,18]$. For instance, if greater self-control affords one increased attentional resources to support ongoing processing of the ethical dilemma and prompt, then the effect of trial type on ITC might decrease as self-control increases. The findings related to ITC may also provide a means to investigate the influence of state variables such as fatigue or stress on the efficiency of processes underpinning decision making in the context of information security [19], as both of these might alter attentional allocation.

The data for the N2 and ITC around $200 \mathrm{~ms}$ after onset of the prompt are consistent with the hypothesis that ethical decision making may be associated with an inward focus of attention. Our previous research reveals that individual differences in self-control, but not moral potency, are related to the amplitude of the N2 in the ISP with the N2 effect being larger in those individuals with low self-control [5]. These and the current findings together lead to the suggestion that reduced attentional resources related to low self-control may be one factor that could result in poor ethical decision making in the ISP. The individual differences measures related to selfcontrol, grit, and pathological technology use should provide us the opportunity to further examine the relationship between these variables and neural recruitment related to ethical decision making in the ISP. 
There are some limitations of our study that must be considered. First, as is true of all our work with the ISP, we are at best measuring an abstract intention related to hypothetical decision making in the laboratory rather than decision making tied to natural contexts with real outcomes. This limitation might be addressed with advances in data collection supported by low-cost reliable EEG systems that could facilitate data collection in quasi-realistic settings or field studies. Second, the sample included undergraduate students and that were primarily female, limiting the generalizability of the findings. Our initial work using ERPs with the ISP only included males in the studies [3], and the physiology observed in that study is quite similar to what we have reported with samples including primarily females [4,5]. Given this, we do not believe that the gender of the sample undermines our findings, however, it may be worth directly considering gender effects in future studies. Also related to the gender imbalance of the study, one might wonder whether female participants are able to empathize with the male protagonist (i.e., Josh) in the ISP. We have sought to address this issue in a recent study using the ISP wherein a gender-neutral name was used for the protagonist along with gender neutral pronouns related to others mentioned in the scenarios. The new study also includes a larger sample (i.e., >200 participants) and roughly equal numbers of males and females, so we should be able to explore this issue at least at the behavioral level.

In conclusion, the current findings build upon prior research using ERPs with the ISP. We demonstrate that violation items are not associated with stronger emotion than control items when assessed with a conscious thought probe. We also replicated the effect of violation trials on the amplitude of the posterior N2 and demonstrated that this effect likely results from differences in phase locking in the Theta/Alpha band rather than differences in overall power between violation and control trials. These findings provide support for the idea that ethical decision making in the ISP may be associated with an inward focus of attention. We believe that the current findings provide a foundation to examine the interplay between individual differences and attentional allocation in the ISP that may serve to bolster or impede ethical decision making. 


\section{References}

1. Richardson, R.: CSI Computer Crime and Security Survey. http://www.GoSCI.com (2011)

2. Cram, W.A., D’Arcy, J., Proudfoot, J.G.: Seeing the Forest and the Trees: A Meta-analysis of the Antecedents to Information Security Policy Compliance. Manag. Inf. Syst. Q 43, 525-554 (2019)

3. $\mathrm{Hu}, \mathrm{Q}$., West, R., Smarandescu, L.: The role of Self-control in Information Security Violations: Insights From a Cognitive Neuroscience Perspective. J Manage Inform Syst 31, 6-48 (2015)

4. Kirby, B., Malley, K., \& West, R.: Neural Activity Related to Information Security Decision Making: Effects of Who is Rewarded and When the Reward is Received. In: F. D. Davis et al. (eds.) Information Systems and Neuroscience, LNISO, 29, 19-27 (2019)

5. West, R., Budde, E., Hu, Q.: Neural Correlates of Decision Making Related to Information Security: Self-control and Moral Potency. PLoS ONE 14(9): e0221808 (2019)

6. Luck, S., Hillyard, S.: (2014). Electrophysiology of Visual Attention in Humans. In: The Cognitive Neurosciences $5^{\text {th }}$ Edition, pp. 187-196 (2014)

7. Andersen, S., Müller, M. Behavioral Performance Follows the Time Course of Neural Facilitation and Suppression During Cued Shifts of Feature-selective Attention. Proc Natl Acad Sci USA 107, 13878-13882 (2010)

8. West, R., Bowry, R., Krompinger, J.: The Effects of Working Memory Demands on the Neural Correlates of Prospective Memory. Neuropsychologia 44, 197-207 (2006)

9. Greene, J., Sommerville, R., Nystrom, L., Darley, J., Cohen, J.: An fMRI Investigation of Emotional Engagement in Moral Judgment. Science, 293, 2105-2108 (2001)

10. Greene, J., Nystrom, L., Engell, A., Darley, J., Cohen, J.: The Neural Bases of Cognitive Conflict and Control in Moral Judgment. Neuron, 44, 389-400 (2004)

11. Duckworth, A., Quinn, P.: Development and validation of the Short Grit Scale (Grit-S). Journal of Personality Assessment, 91, 166-174 (2009)

12. Easton, W, Smith, C., Ybarra, M., Muntaner, C., Tien, A.: Center for epidemiologic studies depression scale: Review and revision (CESD and CESD-R). In: M. E. Maruish (ed.) The use of psychological testing for treatment planning and outcomes assessment: Instruments for adults (pp. 363-377). Lawrence Erlbaum Associates Publishers (2004) 
13. Pawlikowski, M., Alstötter-Gleich, C., Brand, M.: Validation and psychometric properties of a short version of Young's Internet Addiction Test. Comput. Hum. Behav. 29, 1212-1223 (2013)

14. Kwon, Min., Kim, D., Cho, H., Yang, S.: The Smartphone Addiction Scale: Development and Validation of a Short Version for Adolescents. PLOS ONE 8(12) e83558 (2013)

15. Delorme, A., Makeig, S.: EEGLAB: An Open Source Toolbox for Analysis of Single-Trial EEG Dynamics. J. Neurosci. Meth. 143, 9-21 (2004)

16. Lopez-Calderon, J., Luck, S.J.: ERPLAB: An Open-source Toolbox for the Analysis of Event-related Potentials. Front. Hum. Neurosci. 8, 213 (2014)

17. Xu, Z., Hu, Q., Zhang, C.: Why Computer Talents Become Computer Hackers. Comm. ACM. 56, 64-74 (2013)

18. Hu, Q., Zhang, C., Xu, Z.: Moral Beliefs, Self-control, and Sports: Effective Antidotes to the Youth Computer Hacking Epidemic. Paper presented at $45^{\text {th }}$ Hawaii International Conference on Systems Science (2012)

19. Riedl, R.: On the Biology of Technostress: Literature Review and Research Agenda. The DATA BASE for Advances in Information Systems, 44, 18-55 (2012) 